Running Head: THOUGHT EXTRAMISSION

\title{
Thought Extramission: The Eyes Project Conceptual Maps at Close Distance
}

\author{
Magda L. Dumitru ${ }^{*}$ and Gitte H. Joergensen ${ }^{2}$ \\ ${ }^{1}$ Department of Biological and Medical Psychology, University of Bergen, Norway \\ ${ }^{2}$ School of Psychology, University of Connecticut, Mansfield CT, US
}

Corresponding Author:

Dr. Magda L. Dumitru, Department of Biological and Medical Psychology, Jonas Liesvei 91, 5009, University of Bergen, Norway

Email: magda.dumitru@gmail.com 


\begin{abstract}
Light extramission is a popular yet erroneous model of human vision positing that light projects through the eyes over surrounding objects, allowing them to become visible.

However, light extramission research has failed to distinguish between light and other types of emissions coming from the eyes, thereby discounting the possibility that extramission mechanisms might hold true for phenomena other than light. Here, we explored the thought extramission model, which is the notion that thoughts emanate from the eyes in the form of visualized representations. We show that the eyes project thoughts a short distance away in the form of high-resolution conceptual maps whose structure collapses at great distances. We also demonstrate that sensory filtering of thought projection by reducing light contrast lowers the resolution of conceptual maps. Our results highlight the supporting role of the visual system for thought processes relying on contrast, luminance, and perspective formation.
\end{abstract}

Keywords: thought extramission; vision; sunglasses; conceptual processing; conceptual map 


\section{Introduction}

Light extramission is a model of visual perception which has enjoyed a distinguished tradition in the history of science, from the Greek philosophers Croton, Ptolemy, and Euclid to scholars all through the Middle Ages and beyond (Gross 1999). The model assumes that the eyes are a primary source of light projecting over external objects, and is currently the most common explanation of how vision happens among children (Piaget 1926, 1971) and adults (Cottrell \& Winer 1994; Winer \& Cottrell 1996; Winer et al. 1996; Winer et al. 2002) despite having long been replaced by the light intramission model, which accurately describes external light bouncing off objects before entering the eyes. The reason why beliefs in light extramission are still widespread goes beyond undisputed evidence that the eyes harbor internal light, namely the phenomenon referred to as "phosphenes", which are sensations of light in the absence of any external sources (Grüsser \& Hagner 1990; Schutter \& Hortensius 2010). The popularity of the light extramission model is mostly warranted by an extraordinarily persistent belief across human cultures that the eyes radiate some form of invisible energy or magical force.

Although there is no scientific evidence of energy coming from the eyes that (negatively) impacts people or objects, there is experimental evidence that the direction of gaze is coupled with the orienting of attention (Deubel \& Schneider 1996; Friesen \& Kingstone 1998; Hoffman \& Subramaniam 1995), which suggests that eye movements reflect volitional control. Thus, when asked to saccade to a target and report the identity of either the target or a neighbouring distractor, individuals can promptly shift their gaze to a particular location if and only if they are also attending to it, which suggests that visuospatial attention regulates saccadic eye movements. The association between eye-gaze and conscious thought is particularly robust, as people continue to follow specific spatial indices on an empty screen corresponding to actual objects 
from a previously watched visual scene (Altmann 2004; Ferreira, Apel, \& Henderson 2008;

Johansson et al. 2006; Laeng \& Teodorescu 2002; Richardson \& Spivey 2000). In other words, the visual system helps keep track of objects, their properties, and their locations by generating and re-enacting coherent representations of the external world kept in short-term memory. The eyes are also engaged when imagining objects or when retrieving object information from long term memory. As shown in a recent study (Mathot, Grainger \& Strijkers 2017), word-evoked concepts trigger involuntary physiological responses, such that eye pupils become smaller when people read or hear words that convey brightness, than when they read or hear words that convey darkness. Here, we investigated how individuals relate to the objects corresponding to concepts in their mind's eye by testing what we call the "thought extramission model", which is the notion that thoughts emanate from the eyes in the form of visualized representations.

Language is instrumental in the formation of conceptual representations, which differ from short-term memories of visual objects. Indeed, conceptual maps are not directly derived from physical maps via repeated exposure to visual objects and situations, which would ultimately ensure their storage as conceptual structures in long-term memory. Specifically, individuals who are unable to perceive (certain) colors, were reportedly successful in assessing the similarity between two colors that were simply being mentioned and not directly perceived (adequately), by relying on internal conceptual maps (Shepard \& Cooper 1992). Concepts or natural classes can be defined as regions in psychological space that are ultimately determined by their evolutionary relevance. Thus, invariance in psychological space is achieved across perceptual dimensions as generalizations over stimuli sets irrespective of evolutionary-irrelevant variations in their physical properties (Shepard 1987). For example, each colour of the spectrum is constructed by a certain combination of hue, saturation, and luminance, yet a universal 
psychophysical function can be defined that maps physical parameter space onto psychological space by generalizing across the psychological and not merely the psychometric distance between stimuli. The function is monotonically decreasing that is, it decays exponentially with increasing psychological distance between stimuli perceptual attributes.

We tested the thought extramission model by attempting to define a psychological function over the size of objects recorded in long-term memory under several sensory conditions. In a previous study (Dumitru \& Joergensen 2015), we asked participants to evaluate object size or object similarity for homogeneous groups evoked by words read off a computer screen (e.g., 'rabbits' or 'elephants') and showed that objects of the same kind are evaluated as being more dissimilar to each other the bigger they are considered to be. The significant correlations we obtained between objects' size and objects' similarity demonstrate that concepts are organised as conceptual maps, hence as stable representations in the mind's eye. Here, we collected size and similarity evaluations from participants who were asked to visualize objects situated either closeby or far-away from an invariant egocentric location. We aimed to determine whether individuals project conceptual maps at close distance as well as at far distance by computing correlations between size and similarity estimations at each particular distance. We also aimed to determine whether estimation scores in each size condition are equal to those obtained in the default condition, which would directly inform us on whether the canvass on which thought images are projected is situated by default at a certain distance in front of the eyes. Further, we contrasted size and similarity estimations in conditions of high and low visual acuity by asking half of the participants to wear sunglasses. Sunglasses straightforwardly lower the amount of light entering the eyes, thereby allowing to establish whether conceptual representations are ultimately sensitive to the quality of visual perception. 

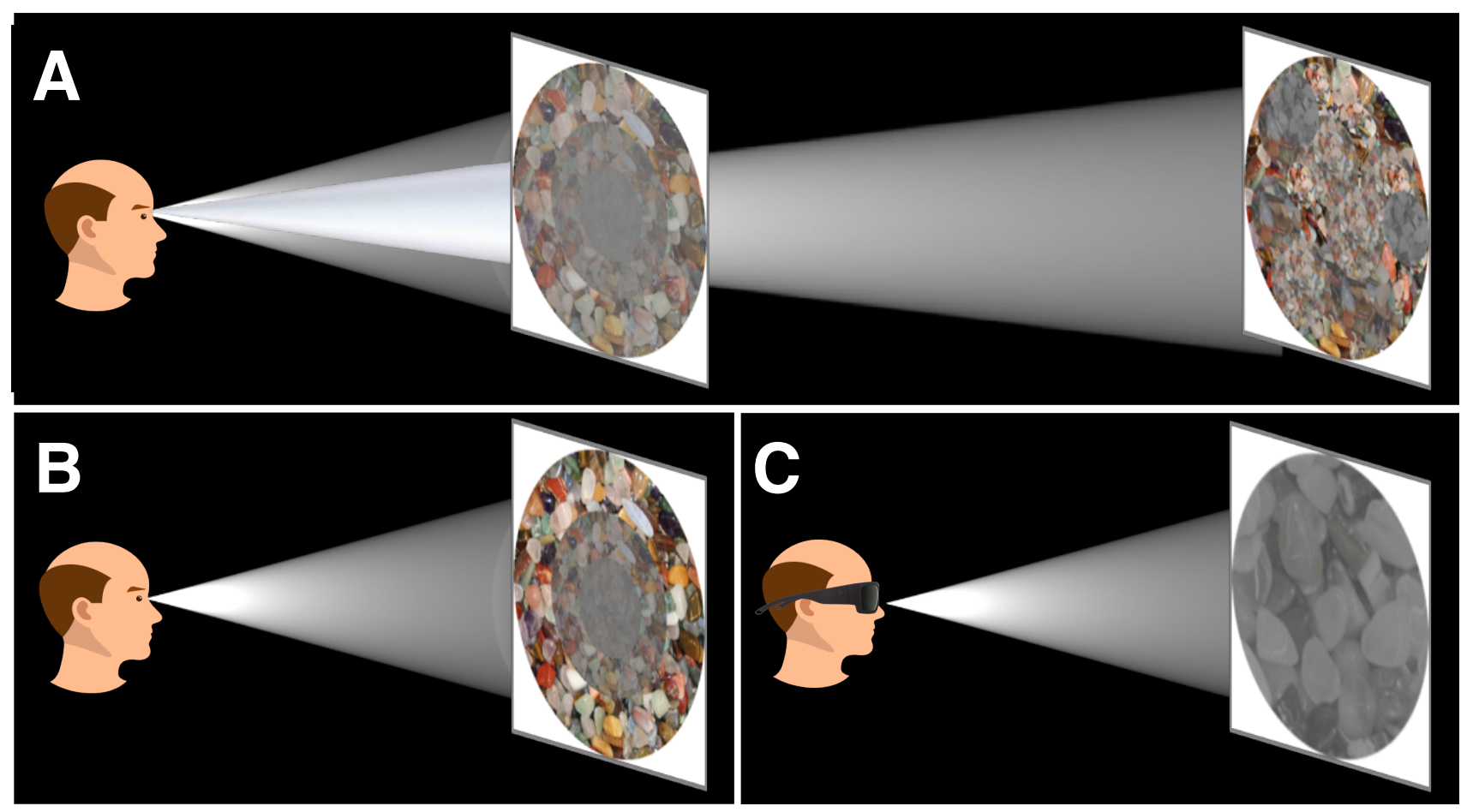

Fig. 1. An illustration of the thought extramission model. Individuals project through their eyes conceptual representations evoked by the word "stone", "pebbles", and "sand", whose decreasing size scores correlates with increasing similarity scores in the default condition (B), close-up condition (A), and sunglasses condition (C), but not in the far-away condition (A). Big, medium, and small size representations are rendered here, for convenience, in ever smaller concentric circles. Conceptual maps have higher resolution in the default condition (B) than in the close-up condition (A).

\section{Methods}

\section{Participants}

A total of 360 volunteers with normal or corrected-to-normal vision and English as their mother tongue participated in the first study. For the second study, we recruited 180 volunteers in the 
same conditions. All volunteers were students at the University of Connecticut and signed an informed consent form upon enrolment in the study, in accordance with the Declaration of Helsinki. They received course credit (pro rata) for their participation in testing sessions lasting up to 60 minutes. The protocol for both studies was approved by the Ethics Committee of The University of Connecticut.

\section{Stimuli}

In both studies, word stimuli (Brysbaert \& New 2009) were drawn from the conceptual category of animals, plants, or objects, were individually randomized, and were each presented onscreen for participants to evaluate. There were 90 stimuli used in the first study and 274 stimuli used in the second study. For both studies, the same set of stimuli was presented for each group of participants that is, for both size and similarity rating tasks.

\section{Design and procedure}

In the first study, we contrasted the experimental conditions close-up, far-away, and default relative to where participants would imagine various objects to be situated, which were mentioned by words appearing onscreen. In the second study, we contrasted size and similarity ratings by participants wearing sunglasses and participants not wearing sunglasses.

Upon completing the participation consent form, we randomly assigned participants in the first study to one of six equal groups and we elicited for each group one of two measurements, namely size estimations or similarity estimations, such that the first group rated the size of objects imagined to stand close to the observer, the second group rated the size of objects imagined to stand far from the observer, the third group rated the size of objects as people 
imagine them by default. Further, the fourth group rated the similarity of objects imagined to be standing close to the observer, the fifth group rated the similarity of objects imagined as being far from the observer, and the sixth group rated the similarity of objects as people imagine them by default. In summary, the first study involved a 3 (Object position: close-by vs far-away vs. default) x 2 (Evaluations: size vs. similarity) full factorial between-subjects experimental design.

We randomly assigned the participants in the second study to one of four equal groups for which we elicited, as in the first study, either size or similarity estimations. The first group rated the size of imagined objects, the second group rated the size of imagined objects while wearing sunglasses, the third group rated the similarity of imagined objects, and the fourth group rated the similarity of imagined objects while wearing sunglasses. In summary, the second study involved a 2 (Vision: sunglasses vs. default) x 2 (Evaluations: size vs. similarity) full factorial between-subjects experimental design.

All participants were seated at a comfortable distance in front of a computer screen and were asked to carefully read each word stimulus presented one by one, and respond by moving a cursor horizontally using the mouse.

\section{Results}

For the first study, we performed a one-way ANOVA over size ratings across the three size conditions (close-up, far-away, and default) and a one-way ANOVA over the three corresponding similarity conditions. Each analysis was followed by Bonferroni-corrected paired comparisons. For the second study, we performed independent-sample $t$-tests for size estimations and for similarity estimations contrasting the sunglasses and the default conditions. 
When comparing close-by, far-away, and default conditions (Fig. 2), we found significant differences for size estimations $[\mathrm{F}(2,177)=5.62, p=0.004]$, such that the objects were evaluated as being smaller when projected far-away than when projected close-by $(p=0.004)$ or by default $(p=0.005)$, as seen in Figure 2. Objects appeared to have about the same size when projected close-by and by default $(p=0.682)$. For similarity estimations, we found significant differences between the three conditions $[\mathrm{F}(2,177)=5.87, p=0.003]$, with objects evaluated as being less similar to each other when projected by default than when projected close-by ( $p=$ 0.007 ) or far-away $(p=0.015)$. Objects appeared equally similar when projected close-by and far-away $(p=0.969)$. Size and similarity item scores were significantly correlated in the close-by condition $(r=-0.400, p<0.001)$ and in the default condition $(r=-.235, p=0.025)$, but not in the far-away condition $(r=-.160, p=0.132)$. 

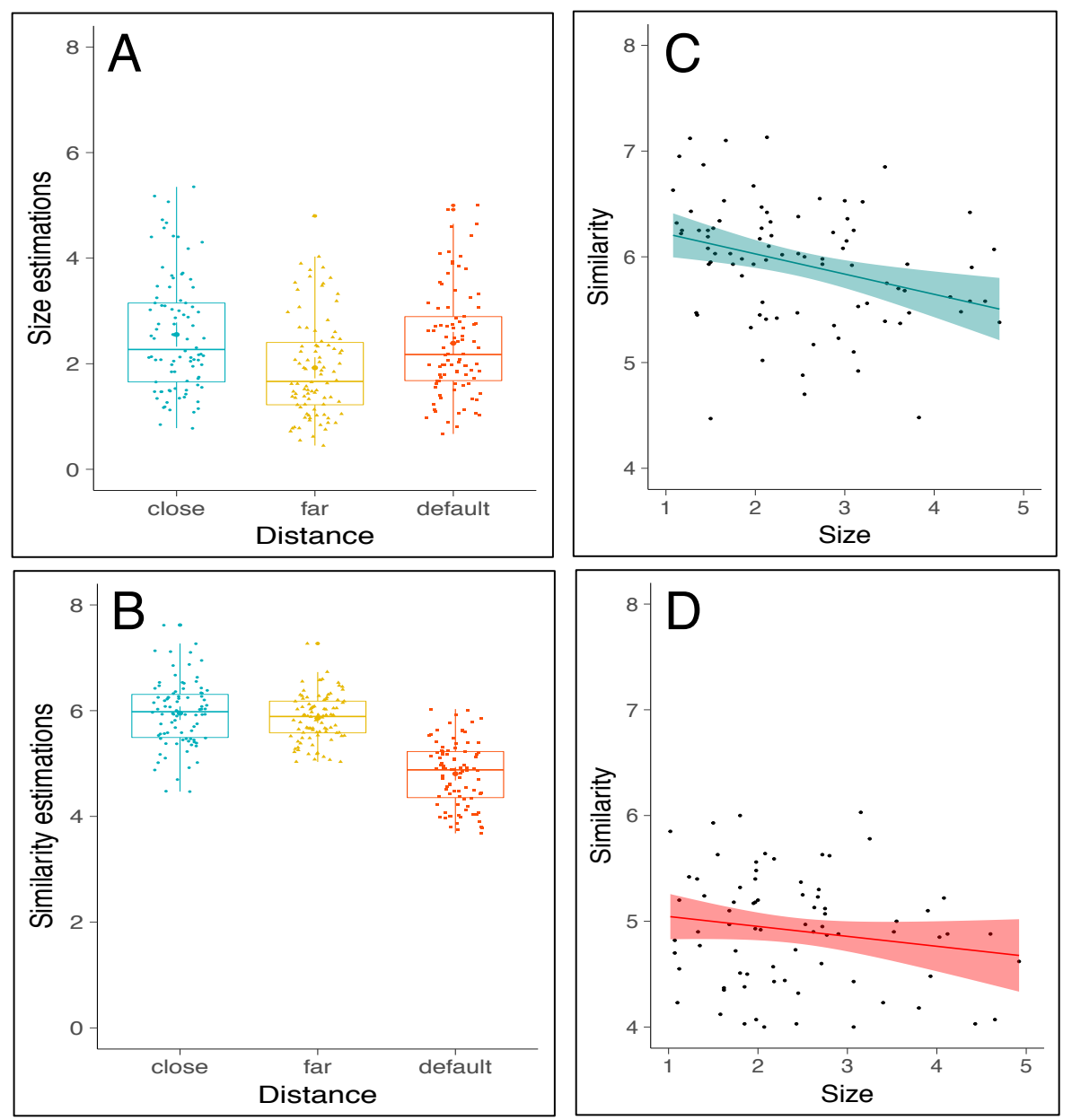

Fig. 2. Summary of results for the first study. Average object size estimations (A) and object similarity estimations (B) across conditions. Boxplots represent scores median, first and third quartiles, minima and maxima. Size and similarity scores correlate significantly with each other in the close-up condition (C) and in the default condition (D) but not in the far-away condition, suggesting that conceptual maps collapse at great distances. Shaded area represents 95\% C.I.s.

In the second study contrasting sunglasses and control conditions (Fig. 3), we found that objects appeared larger $[t(88)=-1.895, p=0.061]$ and were also more similar to each other $[t(88)$ $=-3.123, p=0.002]$ when individuals wore sunglasses compared to controls. Size and similarity 
item scores correlated significantly in the sunglasses condition $(r=-.177, p=0.003)$ and in the control condition $(r=-.246, p<0.001)$.
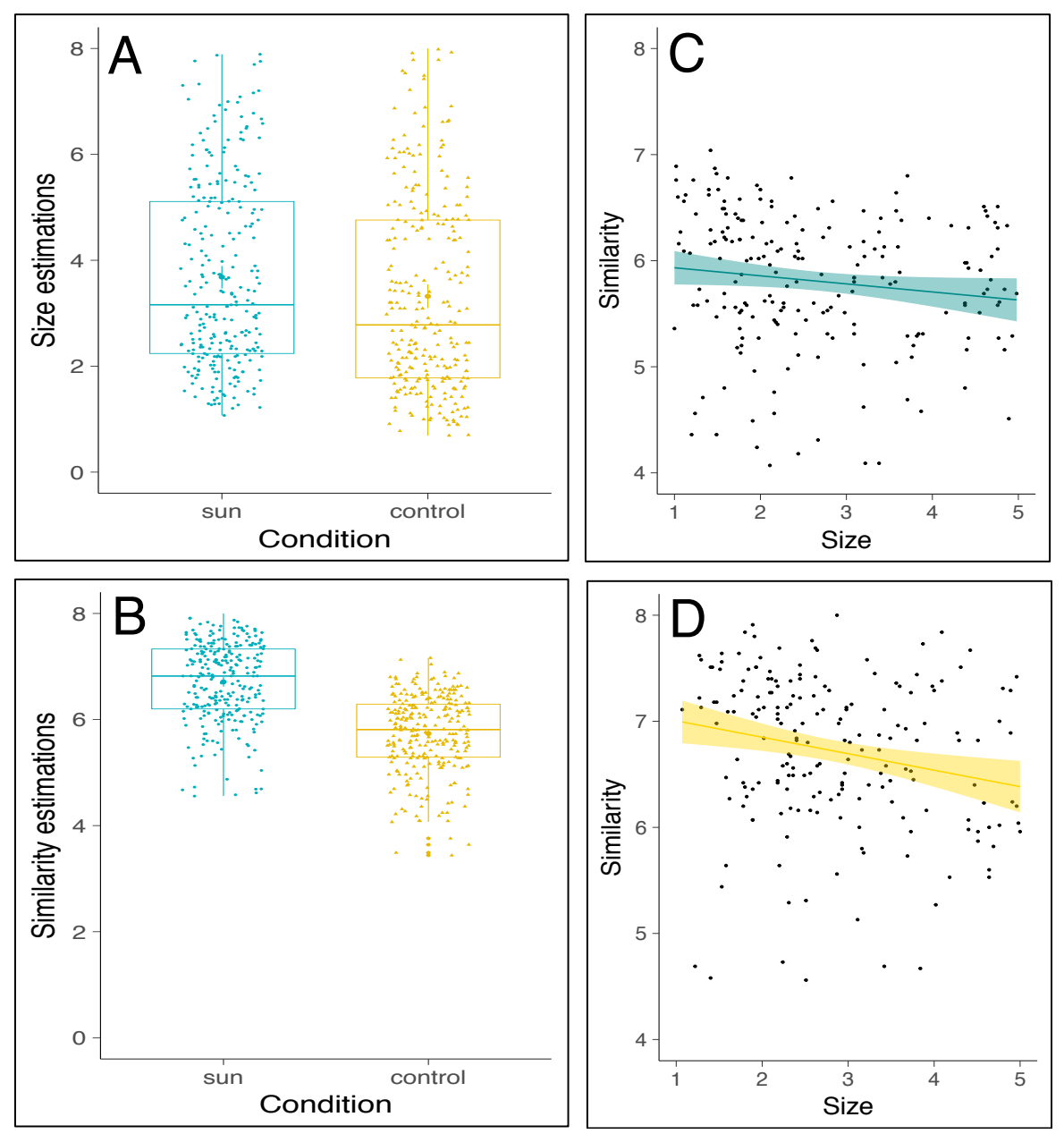

Fig. 3. Summary of results for the second study. Average object size estimations (A) and average object similarity estimations (B) across conditions. Boxplots represent scores median, first and third quartiles, minima and maxima. Size and similarity scores correlate significantly with each other in the sunglasses condition (C) and in the control condition (D), suggesting that object representations form conceptual maps. Shaded area represents 95\% C.I.s. 


\section{Discussion}

We have demonstrated that individuals project conceptual maps by default, at close distance, and while wearing sunglasses, as we found significant correlations between size and similarity estimations in all these conditions. However, we found that conceptual maps collapse when projected at great distances, as size-similarity correlations were not significant in the far-away condition. The results also support the conclusion that conceptual maps have higher resolution than imaginary maps projected at an equal distance, as object similarity was lower in the default than in the close-up condition, confirming previous findings that mental representations evoked by language are qualitatively different from mental images (e.g., Shepard \& Cooper 1992). In particular, the authors reported that color-deficient subjects experience mental images of red and green hues as being more vivid and distinct from each other than the color sensations evoked by the actual red and green objects they were actually seeing. Interestingly, conceptual maps projected through sunglasses, whose resolution was lower than that of default maps (i.e., they generated an increase in similarity estimations) were associated to an increase in size estimations, which we attribute to the activation of common cortical structures when processing object size and/or luminance (Pinel, Piazza, \& Le Bihan 2004).

Our findings have important implications for various interpretations of theories of embodied cognition. In particular, we have shown that words trigger image monitoring through eye gaze in a way that is similar to direct sensory perception of visual objects. Moreover, projecting objects in the mind's eye at close distance by default might be necessary for ensuring the predictability for these representations. Given that projecting images of objects at great distances is unreliable, leading to a disintegration of conceptual maps, all attempts to build action plans based on such representations formed in the mind's eye would become ineffective. Alternatively, there might 
be too much effort required to project images at great distance, similar to trying to maintain high visual acuity when monitoring objects situated far away, which in turn might favour elaboration of conceptual maps at close distance to spare energy resources. Yet another possible explanation for the propensity to project conceptual maps at close distance, which is also motivated by evolutionary purposes, is that human attention is preferentially directed towards objects situated in the immediate proximity of one's body because phenomena which are rather distal are unlikely to form an immediate threat, and ought to be disregarded.

Light extramission has been studied for being a widespread model of visual perception, and this investigation has been mostly undertaken in explicit tasks, which raises methodological concerns. For example, Winer, Cottrell and colleagues asked college students to choose among several vision models simultaneously presented on a computer screen and depicting various combinations of rays, waves, or energy entering or exiting the eyes when people see. The overwhelming proportion of participants who reportedly endorsed light extramission was only mitigated in tasks requiring them to draw lines between an object and a face profile on repeated instances, or in tasks where the objects were themselves producing light (e.g. a light bulb). By asking subjects to decide on a model of vision while failing to distinguish between light and other types of emissions coming from the eyes conflates beliefs in light extramission with beliefs in mechanisms that may hold true for phenomena other than light, such as waves emanating from the eyes that have magical powers (e.g., "the evil eye") or bear emotional valence (e.g., threat and affection), or yet eye emissions in the form of thoughts accompanying visual processes. Our results help explain the erroneous but widespread beliefs in light extramission by showing that information is projected through the eyes, albeit not in the form of light particles. The evidence we provided for thought extramission - that conceptual maps project through the eyes - is not far 
from Plato's idea that emissions from the eyes are triggered by and combine inextricably with daylight before landing on visual objects1. Indeed, thinking and seeing are both voluntary acts of orienting towards objects outside one's own body, and our findings that they influence each other suggest that they may work together. 


\section{References}

Altmann, G. T. (2004). Language-mediated eye movements in the absence of a visual world: the ‘blank screen’ paradigm. Cognition, 93, B79-B87.

Brysbaert, M. \& New, B. (2009). Moving beyond Kucera and Francis: A critical evaluation of current word frequency norms and the introduction of a new and improved frequency measure for American English. Behavior Research Methods, 41, 977-990.

Cottrell, J. E., \& Winer, G. A. (1994). Development in the understanding of perception: The decline of extramission perception beliefs. Developmental Psychology, 30, 218-228.

Deubel, H., \& Schneider, W.X. (1996). Saccade target selection and object recognition: evidence for a common attentional mechanism. Vision Research, 36 (12), 1827-1837.

Dumitru, M. L., \& Joergensen, G. H. (2015). Similarity judgments of same-category object representations: Effects of physical size, manipulability, and word frequency. Visual Cognition, 23 (7), 855-859.

Friesen, C. K., \& Kingstone, A. (1998). The eyes have it! Reflexive orienting is triggered by nonpredictive gaze. Psychonomic Bulletin and Review, 5, 490-495.

Ferreira, F., Apel, J., \& Henderson, J. (2008). Taking a new look at looking at nothing. Trends in Cognitive Sciences, 12 (11), 405-410.

Gross, C. G. (1999) The fire that comes from the eye. The Neuroscientist 5, 58-64.

Hoffman, J.E., \& Subramaniam, B. (1995). The role of visual attention in saccadic eye movements. Perception and Psychophysics, 57(6): 787-795.

Johansson, R. et al. (2006). Pictures and spoken descriptions elicit similar eye movements during mental imagery, both in light and incomplete darkness. Cognitive Science 30, 1053-1079. 
Laeng, B., \& Teodorescu, D.S. (2002). Eye scanpaths during visual imagery re-enact those of perception of the same visual scene. Cognitive Science, 26, 207-231.

Grüsser, O. J., \& Hagner, M. (1990). On the history of deformation phosphenes and the idea of internal light generated in the eye for the purpose of vision. Documenta Ophthalmologica. $74(1-2), 57-85$.

Mathot, S., Grainger, J., \& Strijkers, K. (2017). Pupillary responses to words that convey a sense of brightness or darkness. Psychological Science, 28 (8), 1116-1124.

Piaget, J. (1926). La représentation du monde chez l'enfant, Félix Alcan.

Piaget, J. (1971). Les explications causales, Paris, PUF.

Pinel, P., Piazza, M., \& Le Bihan, D. (2004). Distributed and overlapping cerebral representations of number, size, and luminance during comparative judgments. Neuron, 41, 963-993.

Richardson, D. C., \& Spivey, M. J. (2000). Representation, space and Hollywood Squares: looking at things that aren't there anymore. Cognition 76, 269-295.

Schutter, D. J., \& Hortensius, R. (2010). "Retinal origin of phosphenes to transcranial alternating current stimulation". Clinical Neurophysiology, 121, 1080-1084.

Shepard, R. N. (1987). Toward a universal law of generalization for psychological science. Science, 237, 1317-1323.

Shepard, R. N., \& Cooper, L. A. (1992). Toward a universal law of generalization for psychological science. Psychological Science, 3(2), 97-104.

Spivey, M. J., \& Geng, J. J. (2001). Oculomotor mechanisms activated by imagery and memory: eye movements to absent objects. Psychological Research, 65, 235-241. 
Winer, G. A., Cottrell, J. E., Karefilaki, K. D., \& Gregg, V. R. (1996). Images, words, and questions: Variables that influence beliefs about vision in children and adults. Journal of Experimental Child Psychology, 63, 499-525.

Winer, G. A., Cottrell, J. E., Gregg, V., Fournier, J. S., \& Bica, L. A. (2002). Fundamentally misunderstanding visual perception. Adults' belief in visual emissions. American Psychologist, $57,417-424$.

Winer, G. A., \& Cottrell, J. E. (1996). Effects of drawing on directional representations of the process of vision. Journal of Educational Psychology, 88, 704-717. 